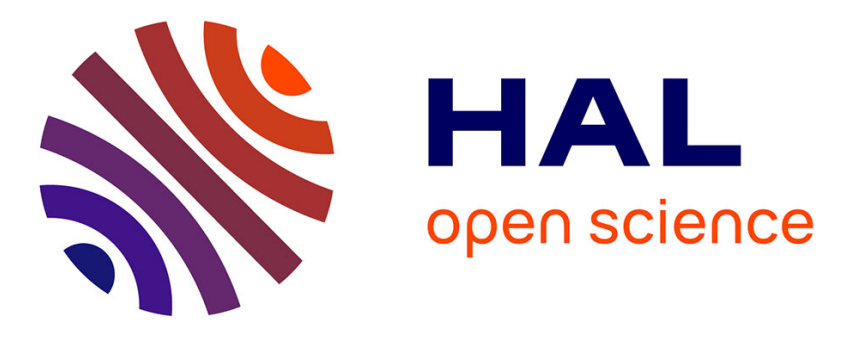

\title{
Brain connectivity changes during ictal aggression (a strangulation attempt)
}

Fabrice Bartolomei, Stanislas Lagarde, Isabelle Lambert, Agnès Trébuchon, Samuel Médina Villalon, Aileen Mcgonigal, Christian Bénar

\section{- To cite this version:}

Fabrice Bartolomei, Stanislas Lagarde, Isabelle Lambert, Agnès Trébuchon, Samuel Médina Villalon, et al.. Brain connectivity changes during ictal aggression (a strangulation attempt). Epileptic Disorders, 2017, 19 (3), pp.367-373. 10.1684/epd.2017.0925 . hal-01851657

\section{HAL Id: hal-01851657 \\ https://hal.science/hal-01851657}

Submitted on 21 Mar 2020

HAL is a multi-disciplinary open access archive for the deposit and dissemination of scientific research documents, whether they are published or not. The documents may come from teaching and research institutions in France or abroad, or from public or private research centers.
L'archive ouverte pluridisciplinaire HAL, est destinée au dépôt et à la diffusion de documents scientifiques de niveau recherche, publiés ou non, émanant des établissements d'enseignement et de recherche français ou étrangers, des laboratoires publics ou privés. 


\title{
Brain connectivity changes during ictal aggression (a strangulation attempt)
}

\author{
Fabrice Bartolomei, Stanislas Lagarde, Isabelle Lambert, \\ Agnes Trébuchon, Samuel Médina Villalon, \\ Aileen McGonigal, Christian G Benar
}

Aix Marseille University, Institut de neurosciences des systèmes and AP-HM, Hôpital de la Timone, Service de neurophysiologie clinique, Marseille 13005, France

Received December 26, 2016; Accepted June 07, 2017

\begin{abstract}
Ictal aggressive behaviour is a rare manifestation of focal seizures. We report an episode of ictal aggression occurring during an intracerebrally recorded seizure (using stereoelectroencephalography) in a patient with drug-resistant temporal lobe epilepsy. Aggression occurred during the last part of the seizure and was coincident with marked EEG slowing of the frontal regions and persistent ictal activity in the medial temporal lobe. A functional connectivity study $\left(\mathrm{h}^{2}\right.$ estimation of interdependencies) showed a bilateral massive hypersynchronization between frontal and temporal regions. This case illustrates the occurrence of aggression during imbalance between the electrical activity in the temporal limbic cortex and prefrontal cortex, in agreement with the current neurobiological theories of aggression.
\end{abstract}

Key words: SEEG, functional connectivity, aggression, focal seizure

Correspondence:

Fabrice Bartolomei

Service de neurophysiologie clinique,

CHU Timone,

264 Rue Saint-Pierre,

13005 Marseille, France

<fabrice.bartolomei@ap-hm.fr>
Minor episodes of aggression are relatively common in patients with epilepsy but they are probably no more frequent than in populations with social disadvantages or brain damage (Rantakallio et al., 1992; Grafman et al., 1996; Treiman, 1999; Tebartz Van Elst et al., 2001; Fazel et al., 2011; Piazzini et al., 2012). Three different forms of aggressive behaviours have been described in epileptic patients on the basis of their temporal relationship with seizures: interictal, post-ictal, and ictal (Tassinari et al., 2005a; Bronsard and Bartolomei, 2013). Of these, postictal aggression, while the patient is in a confused state, is likely to be the most prevalent. However, reported cases with good quality video-EEG recording are rare (Tassinari et al., 2005a) and as a result, it may be difficult to distinguish between true semiological seizure phenomena and postictal confusional behaviour. In seizures affecting brain regions involved in emotion processing, ictal or postictal aggressive behaviour may occur. Defensive-like behaviour can be 
observed, particularly during the immediate postictal period, in which the patient is often confused and in an altered mood state (Delgado-Escueta et al., 1981). In this context, aggressive behaviour is often related to minimally unpleasant stimulation or attempts to restrain the patient (Tassinari et al., 2005a). This phenomenon is rare and has been generally classified as a defensive/aggressive behaviour, related to the interaction between the patient and the caregivers (i.e. nurse during video-EEG monitoring) (Bronsard and Bartolomei, 2013). It is generally thought that the aggressive behaviour is not usually truly dangerous. More serious offences can occur during postictal psychosis, but the clinical picture is close to premeditated/predatory aggression (Kanemoto et al., 2010). The current view of the neurobiology of aggressive behaviour is based on a model of an imbalance between regulatory regions (essentially prefrontal) and regions engaged in emotional and behavioural responses (amygdala, hypothalamus, and brain stem) (Siever, 2008; Rosell and Siever, 2015). Such a scheme has been proposed for seizures associated with the emergence of stereotyped emotional abnormal behaviour, such as rage or aggression (Bartolomei et al., 2005, Tassinari et al., 2005a, 2005b).

Ictal semiology does not depend on the involvement of a unique region but appears to be related to the involvement of a set of regions within specific anatomical systems (Bartolomei et al., 2013). This is particularly true for complex ictal behaviours that necessarily depend upon altered dynamics within widely distributed, rather than focal or regional, neural networks. In this way, considering large-scale network involvement during seizure evolution is a way to investigate the emergence of clinical patterns during seizures. Thus, the study of functional connectivity during seizures has been proposed in the past to be used to quantify the temporo-spatial changes in network properties (Bartolomei et al., 2013).

In the present study, we report a case in which, during one recorded seizures, we observed violent behaviour, clearly occurring during the ictal phase, culminating in an aggressive act with a strangulation attempt. We describe both SEEG aspects and intracerebral EEG connectivity changes. During the aggressive behaviour part of the seizure, major changes in functional connectivity $(\mathrm{Fc})$ occurred between frontal and temporal regions.

\section{Case study}

A 33-year-old male patient underwent presurgical evaluation for his drug-resistant epilepsy in our epilepsy unit. He had had an encephalitic illness at the age 21 , presenting with fever and recurrent seizures in the acute phase. He was treated at this time with acyclovir IV and amoxicillin, and outcome was finally favourable with no neurological or significant cognitive deficit. Seizures developed six months later and were resistant to antiepileptic drugs. Non-invasive presurgical evaluation led to the recording of prominent left side fronto-temporal seizures. Psychiatric evaluation did not reveal major personality disorder or an anxio-depressive state. Magnetic resonance imaging (MRI) did not show any structural abnormality. Intracerebral EEG (stereoencephalography [SEEG]) was subsequently performed for presurgical evaluation. Ten electrodes were placed in brain regions according to hypotheses regarding seizure onset and propagation (mainly the temporo-frontal left side), including the right and left temporo-frontal cortices (figure 1A).

\section{Connectivity analysis}

Interdependencies between SEEG signals were estimated with Anywave software (Colombet et al., 2015) which computes a pairwise non-linear regression analysis based on the $\mathrm{h}^{2}$ coefficient. In summary, a piecewise linear regression is performed between each pair of signals, testing all the shifts of one signal relative to the other within a maximum lag. The $\mathrm{h}^{2}$ is the coefficient of determination that measures the goodness of fit of the non-linear regression. The $\mathrm{h}^{2}$ is bounded between 0 (no correlation) and 1 (maximal correlation) (Wendling and Bartolomei, 2001). We used a sliding window of four seconds with an overlap of two seconds, and a maximum delay between signals of $100 \mathrm{~ms}$.

Signals were filtered using the Anywave software (Colombet et al., 2015) (available at http://meg.univamu.fr/wiki/AnyWave), with Butterworth filters of order 4 . We used only a $0.5-\mathrm{Hz}$ high-pass filter for all the analyses.

\section{Graph measures}

For all the selected channels (each channel being a bipolar derivation), we computed all the pairwise $\mathrm{h}^{2}$ values. Between two channels, we selected the higher h2 value between chan1-> chan2 and chan2$>$ chan1. We thus obtained connectivity graphs, with each channel representing a node of the graph, and h2 values the strength of the link between two nodes. We summarized the connectivity graphs with two related graph measures. The first measure, node degrees, first involves thresholding the graphs, and then counting the number of significant links between a given node and the rest of the graph. This has the advantage of removing the links with low connectivity. 
A

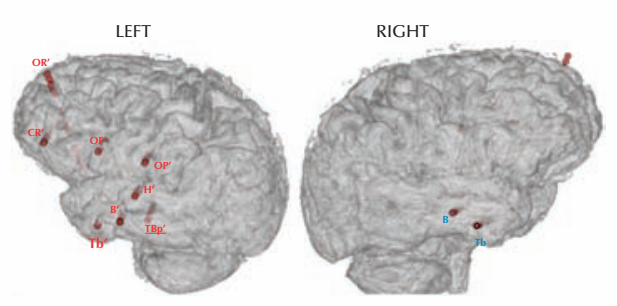

B
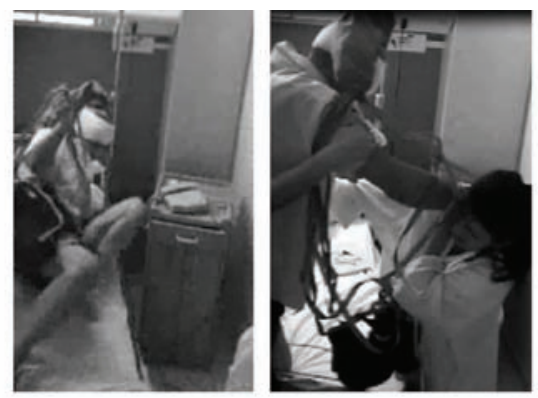

MS

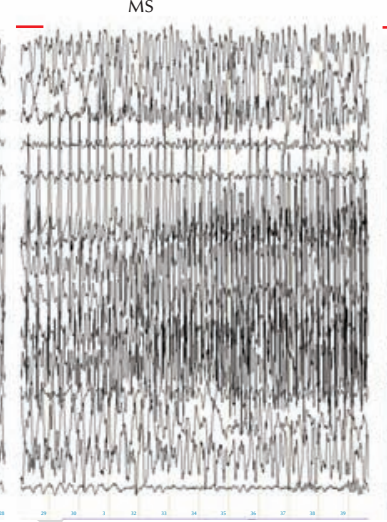

ES

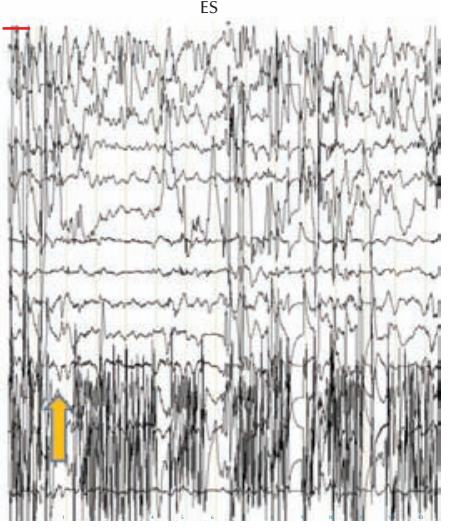

Figure 1. (A) SEEG implantation showing the electrodes on a 3D MRI mesh of the cortex. TB and TB' electrodes were used to investigate the temporo-basal cortex to within the entorhinal cortex; $\mathrm{B}$ and $\mathrm{B}^{\prime}$ electrodes for the anterior hippocampus/amygdala region (medial leads) and the mid part of MTG (lateral leads); $\mathrm{C}^{\prime}$ electrode for the posterior hippocampus and the lateral temporal cortex; $\mathrm{H}^{\prime}$ electrode for the insula (medial leads) and the anterior part of the superior temporal gyrus (STG) (lateral leads); OF' electrode for the frontal operculum; $\mathrm{OP}^{\prime}$ electrode for the parietal operculum; $\mathrm{CR}^{\prime}$ electrode for the prefrontal cortex to within the anterior cingulate region; and $\mathrm{OR}^{\prime}$ electrode for the prefrontal cortex (superior leads) and the orbitofrontal cortex (inferior leads). The prime symbol refers to the left side. (B) Captures from the video-SEEG recordings showing the patient jumping from his bed and grabbing the nurse. (C) SEEG traces showing the seizure onset (SO) and a discharge starting from the left temporal neocortex (blue arrow), before spreading (middle seizure $[\mathrm{MS}]$ ) to the suprasylvian cortices $\left(\mathrm{H}^{\prime}, \mathrm{OF}^{\prime}\right)$ and the frontal cortices $\left(\mathrm{OR}^{\prime}\right)$ and affecting the right temporal lobe. The last part (end of seizure [ES]) is characterized by a slowing in the left regions and a burst activity in the right temporal electrodes. L: left; R: right; OFC: orbitofrontal cortex; DLPFC: dorsolateral prefrontal; CG32: cingulate gyrus Brodmann's area 32; OPF: frontal operculum; STG: superior temporal gyrus; TBC: basal temporal cortex; Hip: hippocampus; EC: entorhinal cortex; MTG: middle temporal gyrus.

The threshold was set empirically to 0.25 . The second measure, node strength, consists of the mean $\mathrm{h} 2$ between one node and all the other nodes. It has the advantage of not relying on a threshold that can be difficult to tune.

\section{Statistical analysis}

Node degrees and connectivity strength were compared between a period preceding seizure onset (background and, in particular, the seizure onset) and the period in which the aggression occurred (end of seizure). A comparison was performed using a Wilcoxon non-parametric paired test and a Bonferroni correction was applied.

\section{Video-SEEG and intracerebral EEG connectivity}

During video-SEEG monitoring (after partial reduction of antiepileptic drugs), 10 seizures were recorded. Eight seizures started in the right lateral temporal cortex and two on the left side involving the temporal regions. One episode of ictal aggression was recorded during a left-sided seizure. The seizure began habitually in clinical terms, characterized by loss of contact and mild gestural automatisms. During the course of the seizure (one minute after the onset), a nurse arrived in the room and interacted verbally with the patient in order to carry out the usual ictal examination protocol. At this moment, the patient became 


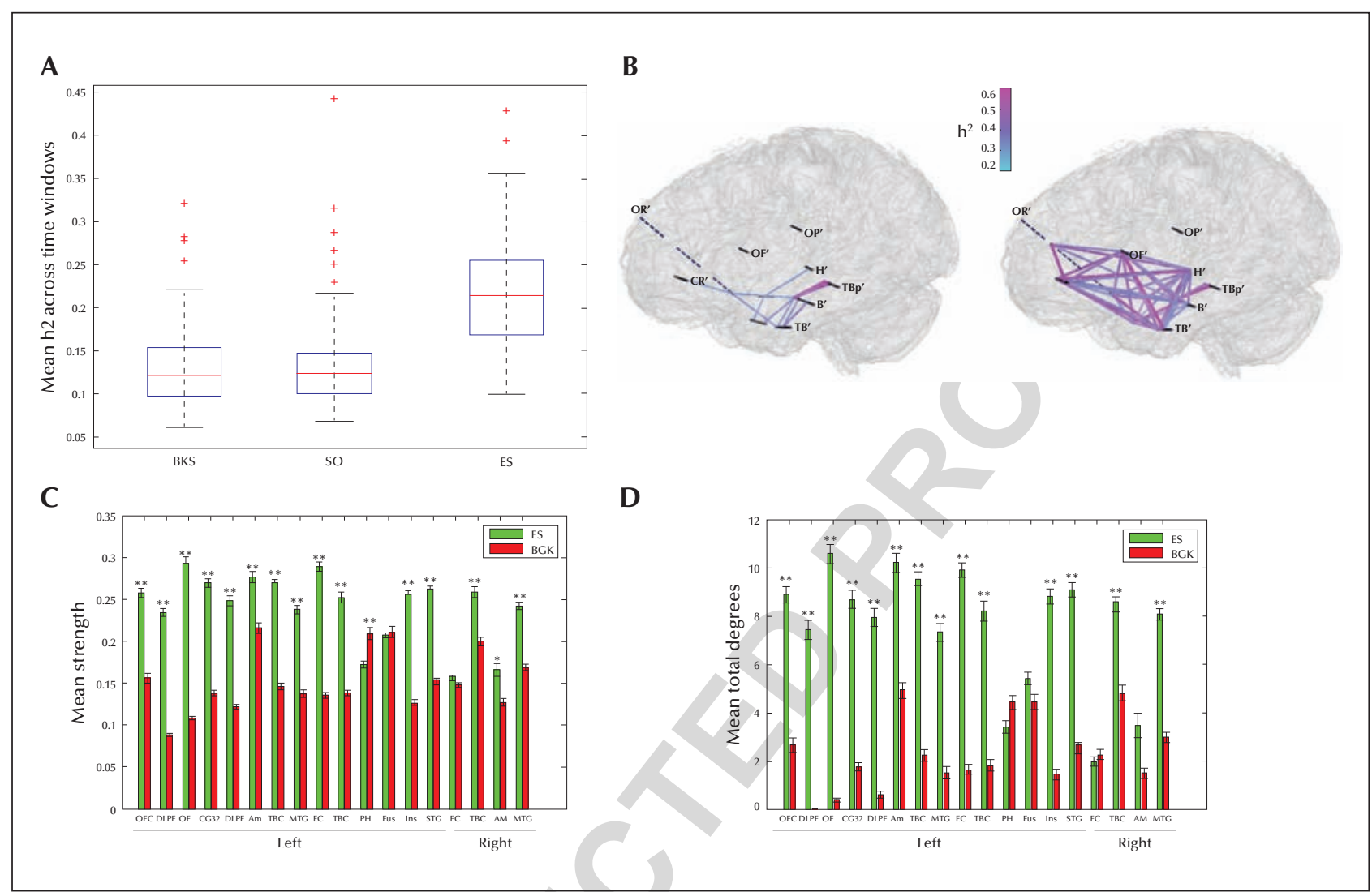

Figure 2. Connectivity analysis. (A) Global changes in connectivity during a seizure with aggression (SO: seizure onset; ES: end of seizure). Mean values over periods of 15 seconds were compared. Changes were significant only for the end of the seizure period $(p<0.00001$ relative to background $[B K G])$. (B) Changes in connectivity are illustrated on a 3D mesh of the MRI with the position of electrodes and the graphs thresholded $\left(h^{2}>0.25\right)$. On the left (seizure onset), the main connected regions are the left temporal contacts, while the end part of the seizure (right) is characterized by connecting links between temporofrontal regions affecting both sides. Colour scale indicates $h^{2}$ values. (C, D) For each selected channel, the mean values of $h^{2}(C)$ and the degrees (D) are indicated between two conditions (background and seizure end period). * significant interactions $(p<0.05$ after Bonferroni corrections).

aggressive, showing combative behaviour with groaning, swearing, and threatening the nurse with his fist. Despite verbal reassurance, the patient then grabbed the nurse and attempted to strangle her (figure 1B). After several seconds and thanks to the intervention of another nurse, the patient stopped and finally returned to his bed. After the seizure, the patient was completely amnesic for the episode.

Electro-anatomo-clinical correlations for this seizure are shown in figure 1. The seizure started from the left temporal region (lateral and medial parts), becoming rapidly bilateral and spreading to the prefrontal cortex. Aggressive behaviour coincided with marked slowing of bilateral frontal region activity occurring in the last part of the seizure and was associated with the persistence of ictal activity under the form of burst activities in the right medial temporal lobe (in particular, the medial contacts of electrodes B which were used to investigate the anterior hippocampus/amygdala region).
A connectivity study (figure $2 B$ ) was performed between 18 bipolar derivations, recording different brain areas, including the two temporal regions and the left prefrontal cortex. This analysis revealed that seizure onset was associated with connectivity changes (an increase) limited to the left temporal regions. The global connectivity was not significantly changed (figure $2 A$ ) at seizure onset $(p=0.8)$, but largely increased at seizure end $(p<0.0001)$. During the aggressive phase (end part of the seizure), a massive change, characterized by increased connectivity, was indeed observed bilaterally, affecting most of the recorded connections between frontal and temporal regions. This hypersynchrony was observed in most of the studied regions, both in terms of strength of connectivity and degrees (figure 2D). We also performed the same analysis during a second temporal seizure for the same patient, starting from the left temporal cortex. In this seizure, despite the same examination by the nurse, no aggression occurred. This seizure started from the left 


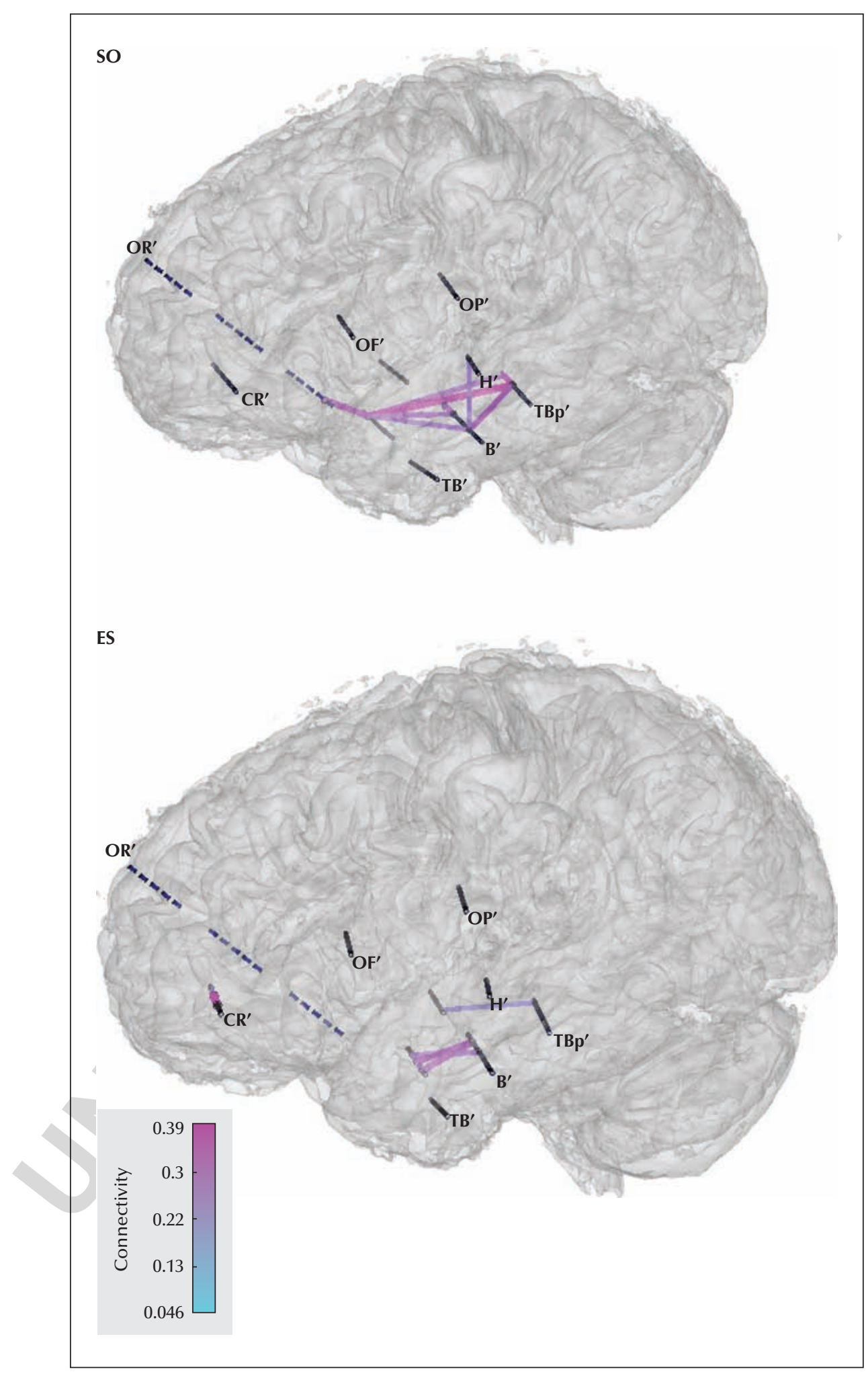

Figure 3. Changes in connectivity during a left side-onset temporal seizure without aggressive behaviour. These are illustrated on a $3 \mathrm{D}$ mesh of the MRI with the position of electrodes and graphs thresholded $\left(h^{2}=0.25\right)$. Colour scale indicates $h^{2}$ values. 
mesial and lateral temporal cortex, spreading to the frontal lobe. This seizure was shorter and the significant frontal lobe slowing observed during the seizure with aggression was not evident. Connectivity changes are indicated in figure 3 and did not show the same pattern of fronto-temporal hypersynchrony.

\section{Discussion}

We report here seizure-related aggressive behaviour that clearly occurred during the ictal phase of a temporo-frontal seizure, recorded using intracerebral electrodes. The clinical changes observed in our case can be classified as defensive/aggressive ictal behaviour, triggered by the verbal interaction between nurse and patient. However, this was associated with a severe aggressive act (strangulation attempt) which is fairly unusual in this context. In agreement with previous reported cases, our patient had seizures involving the temporo-frontal cortices (Tassinari et al., 2005a; Bronsard and Bartolomei, 2013). The failure of "top-down"control systems in the prefrontal cortex to modulate aggressive acts (with a possible triggering stimulus) appears to play an important role in aggressive behaviour (Davidson et al., 2000; Siever, 2008). An imbalance between prefrontal regulatory influences and hyper-responsivity of the amygdala and other limbic regions involved in affective evaluation is a putative mechanism of aggressive human behaviour (Siever, 2008). The observations in the present case could fit well with this model, since the patient's aggressive behaviour was concomitant with persistent epileptic activity in the limbic system (internal temporal region including the amygdala) and massive slowing of cortical prefrontal regions. Connectivity analysis showed that the brain regions investigated, particularly the frontal-temporal connections, were engaged in massive abnormal hypersynchrony. It could be hypothesized that this pathological hypersynchronous state would lead to a breakdown of normal regulatory function of the cortical regions. Similar patterns of long-range hypersynchronous activity changes have been correlated with ictal altered consciousness, in line with the theory that seizure-related hypersynchrony disrupts the "global workspace of consciousness" (Bartolomei and Naccache, 2011). Changes in connectivity between amygdala/mesial temporal regions and the frontal lobes have been described in seizures, including profound alterations in emotional behaviour such as screaming, agitation, and facial expression of terror (Bartolomei et al., 2005). Some of these clinical symptoms have been interpreted as the emergence of "archaic" or innate motor repertoires (Tassinari et al., 2005b), in which impaired higher cortical control would allow "pre-programmed" and essentially subcortically organised motor behaviours to be inappropriately expressed or "released". This idea of hierarchical control of behaviour can indeed be traced back to Hughlings Jackson (Jackson, 1931). In this context, aggressive behaviour, such as biting, is an interesting finding observed in some patients during seizures (Bartolomeil et al., 2002; Tassinari et al., 2005a) that may indeed reflect the stimulation of an innate program because of its similarity to animal behaviours (Tassinari et al., 2005a).

In conclusion, our case is a rare report of intracerebrally recorded ictal aggressive behaviour associated with altered network dynamics within fronto-limbic circuits. Clearly, no firm conclusions can be drawn from analysis of a single seizure, in terms of whether the observed dysfunction within these networks might have an eventual causal role or not. However, the rarity of the observation appears to merit discussion. Current methods of signal analysis of intracerebrally recorded seizures allow estimation of network dynamics correlated with evolving clinical signs, with notably high temporal resolution in comparison to other methods, such as functional MRI. The electrical data obtained is entirely dependent on the recording sites selected according to clinical indication for presurgical evaluation. As such, the vast majority of available data in seizure anatomo-electrical clinical correlation are taken from small-volume samples of cortical and/or limbic structures, albeit precisely defined anatomically and chosen in order to logically sample known networks. Understanding of the neural basis of complex ictal behaviours remains extremely elusive and it can be imagined that in order to make progress in this area, current methods would need to be combined with means of studying dynamic change within more widespread networks, including multiple subcortical structures. $\square$

\section{Supplementary data.}

Summary didactic slides are available on the www.epilepticdisorders.com website.

\section{Acknowledgements and disclosures.}

We thank Pr J Regis for stereotactic procedures and Pr P Chauvel for clinical assessment of the patient.

The authors have no conflict of interest to disclose.

\section{References}

Bartolomei F, Trebuchon A, Gavaret M, Regis J, Wendling F, Chauvel P. Acute alteration of emotional behaviour in epileptic seizures is related to transient desynchrony in emotionregulation networks. Clin Neurophysiol 2005; 116: 2473-9. 
Bartolomei F, Guye M, Wendling F. Abnormal binding and disruption in large scale networks involved in human partial seizures. EPJ Nonlinear Biomed Phys 2013; 1.

Bartolomei F, Naccache L. The global workspace (GW) theory of consciousness and epilepsy. Behav Neurol 2011; 24: 67-74.

Bartolomeil F, Guye M, Wendling F, Gavaret $M$, Regis J, Chauvel P. Fear, anger and compulsive behavior during seizure: involvement of large scale frontotemporal neural networks. Epileptic Disord 2002; 4: 235-41.

Bronsard G, Bartolomei F. Rhythms, rhythmicity and aggression. J Physiol Paris 2013;107:327-34.

Colombet B, Woodman M, Badier JM, Benar CG. AnyWave: a cross-platform and modular software for visualizing and processing electrophysiological signals. J Neurosci Methods 2015; 242: 118-26.

Davidson RJ, Putman KM, Larson CL. Dysfunction in the neural circuitry of emotion regulation: a possible prelude to violence. Science 2000; 289: 591-4.

Delgado-Escueta AV, Mattson RH, King L, et al. Special report. The nature of aggression during epileptic seizures. $N$ Engl J Med 1981; 305: 711-6.

Fazel S, Lichtenstein P, Grann M, Langstrom N. Risk of violent crime in individuals with epilepsy and traumatic brain injury: a 35-year Swedish population study. PLoS Med 2011; 8: e1001150.

Grafman J, Schwab K, Warden D, Pridgen A, Brown HR, Salazar AM. Frontal lobe injuries, violence, and aggression: a report of the Vietnam Head Injury Study. Neurology 1996; 46: 1231-8.
Jackson J. Selected Writing of John Hughling Jackson Vol 1: On epilepsy and Epileptiform Convulsions. London: Holder \& Stoughton, 1931.

Kanemoto K, Tadokoro Y, Oshima T. Violence and postictal psychosis: a comparison of postictal psychosis, interictal psychosis, and postictal confusion. Epilepsy Behav 2010; 19: 162-6.

Piazzini A, Bravi F, Edefonti V, et al. Aggressive behavior and epilepsy: a multicenter study. Epilepsia 2012; 53: e174-9.

Rantakallio P, Koiranen M, Mottonen J. Association of perinatal events, epilepsy, and central nervous system trauma with juvenile delinquency. Arch Dis Child 1992; 67: 1459-61.

Rosell DR, Siever LJ. The neurobiology of aggression and violence. CNS Spectr 2015; 20: 254-79.

Siever LJ. Neurobiology of aggression and violence. Am J Psychiatry 2008; 165: 429-42.

Tassinari CA, Tassi L, Calandra-Buonaura G, et al. Biting behavior, aggression, and seizures. Epilepsia 2005a; 46: 654-63.

Tassinari CA, Rubboli G, Gardella E, et al. Central pattern generators for a common semiology in fronto-limbic seizures and in parasomnias. A neuroethologic approach. Neurol Sci 2005b; 26(3): s225-32.

Tebartz Van Elst L, Ebert D, Trimble MR. Aggression and Violence in Patients with Epilepsy. Epilepsy Behav 2001; 2: 65-6.

Treiman DM. Violence and the epilepsy defense. Neurol Clin 1999; 17: 245-55.

Wendling F, Bartolomei F. Modeling EEG signals and interpreting measures of relationship during temporal-lobe seizures: an approach to the study of epileptogenic networks. Epileptic Disord 2001; Special Issue: 67-78.

\section{TEST YOURSELF}

(1) What is the main form of aggression that may be observed during a temporal or temporo-frontal seizure?

(2) Defensive aggressive behaviour is considered as failure of "top-down" control systems in the prefrontal cortex to modulate aggressive acts. As a consequence, which key structure of the brain could be hyperactive?

Note: Reading the manuscript provides an answer to all questions. Correct answers may be accessed on the website, www.epilepticdisorders.com, under the section "The EpiCentre". 


\section{Author Query}

No query 